\title{
NECROSIS OF ARTICULAR CARTILAGE AFTER SLIPPING OF THE CAPITAL FEMORAL EPIPHYSIS
}

\author{
Report of Six Cases with Recovery
}

\author{
H. G. Lowe, Stafford, Englani)
}

Since Elmslie in 1913 and Waldenström in 1930 described the complication of necrosis of articular cartilage after slipping of the capital femoral epiphysis, it has become gradually recognised as a clinical and pathological entity to be distinguished from avascular necrosis of the bony epiphysis and secondary osteoarthritis. Necrosis of articular cartilage is characterised clinically by increasing stiffness of the hip joint, with muscle spasm causing flexion and adduction contractures proceeding -in severe cases--to fibrous ankylosis. Pain is a variable symptom but it can be mild. Radiographically, there is increasing loss of joint space, and the cortical surfaces of the femoral head and acetabulum become irregular, with osteoporosis of the head of femur and acetabulum. The complication usually arises within the first year after the diagnosis of slipping-sometimes within a few weeks. It can arise spontaneously after slipping of the femoral epiphysis without any treatment and may follow either a slight or a severe slip. It may occur after any type of treatment, whether conservative or operative. The duration of symptoms before the patient seeks advice may be long or short but is usually long. American literature records a relatively high incidence in negro races. There seems to be no sex distinction and puberty usually proceeds normally. The lesion is seen in tall thin children and in fat short children. The epiphysial line proceeds to fusion. In bilateral slipping of the epiphysis both hips may be affected by necrosis of the joint cartilage or one hip alone.

The prognosis after necrosis of articular cartilage following slipping of the capital femoral epiphysis has been thought to be poor, and arthrodesis or arthroplasty has often been carried out. This paper records six cases in which joint space and movement recovered, with good function.

\section{CASE REPORTS}

Case 1-A girl aged eleven first attended hospital complaining of pain in the left groin. One year previously slipping of the opposite (right) femoral epiphysis had been treated by pinning. Radiographs of the left hip showed slight posterior slipping of the epiphysis with a good joint space and normal bone texture (Fig. 1). The epiphysis was secured by insertion of two guide wires. Twelve days after operation she complained of pain in the left hip and did not feel well. The hip was held in 60 degrees of flexion and all movements were painful. There was a sense of fullness in the groin. The temperature was 37.2 degrees Centigrade and the erythrocyte sedimentation rate was 95-116 millimetres per hour. Traction was applied but the hip remained flexed and adducted. The temperature became normal a week later and the sedimentation rate fell to 56-93 millimetres per hour. A plaster hip spica was applied under a general anaesthetic to rest the hip. Later a slight swinging pyrexia recurred. The Rose Waaler test was negative and the white cell count was 7,800 per cubic millimetre.

Radiographs taken six weeks after the pinning operation showed loss of joint space and osteoporosis (Fig. 2). The guide wires were removed because it was thought that infection had occurred, but no evidence of infection was found during their removal, and culture was sterile. After removal of the guide wires the hip was again immobilised in a plaster spica. The temperature continued to swing between 37.2 and 37.8 degrees Centigrade but the erythrocyte sedimentation rate fell to 18-46 millimetres per hour. The hip was explored. At operation the capsule was found to be thickened and the bone of the femoral head and neck 


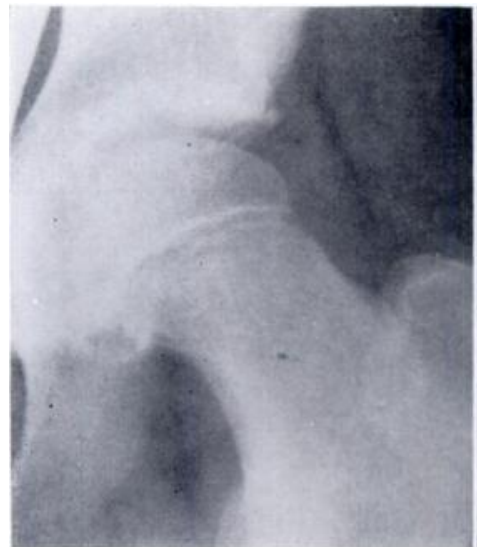

Fig. 1

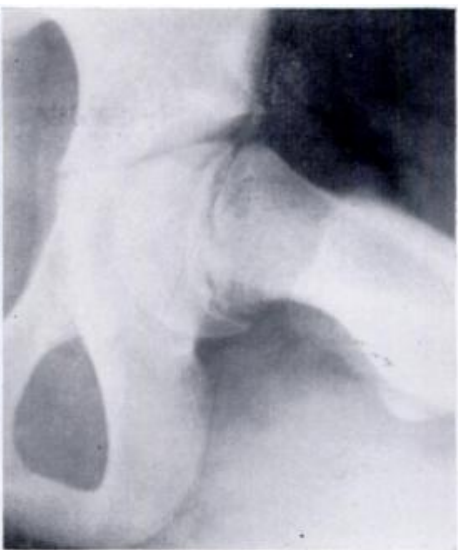

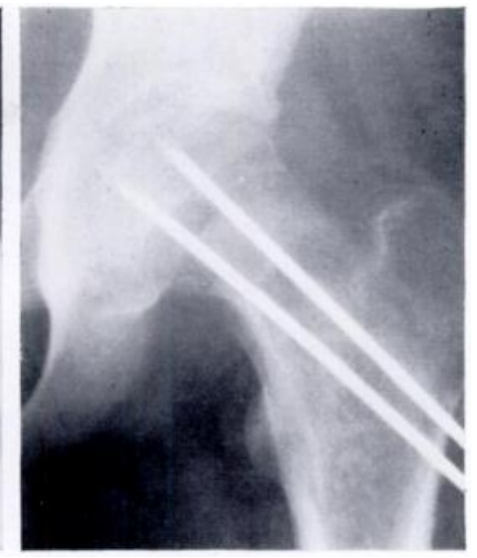

FIG. 2

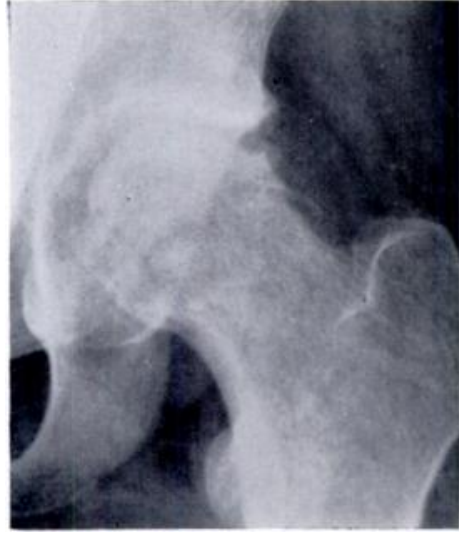

FIG. 3

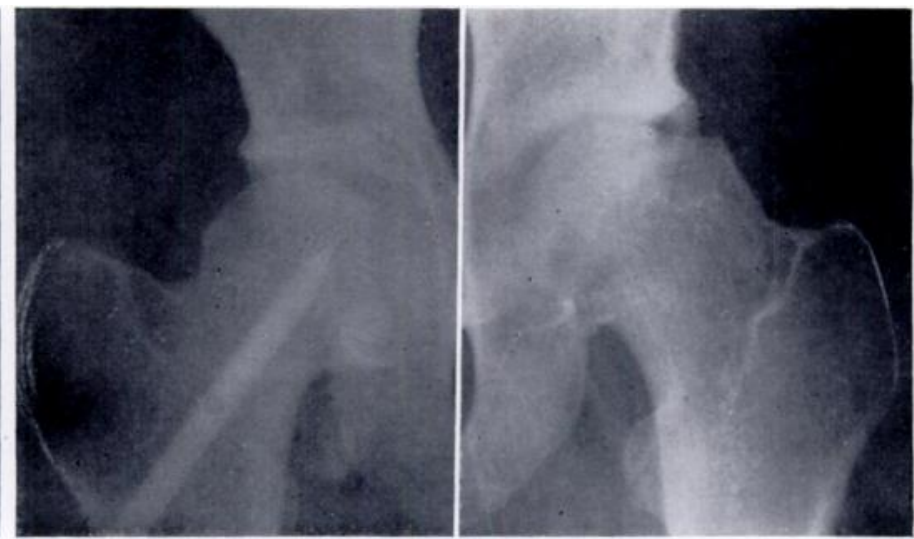

FIG. 4
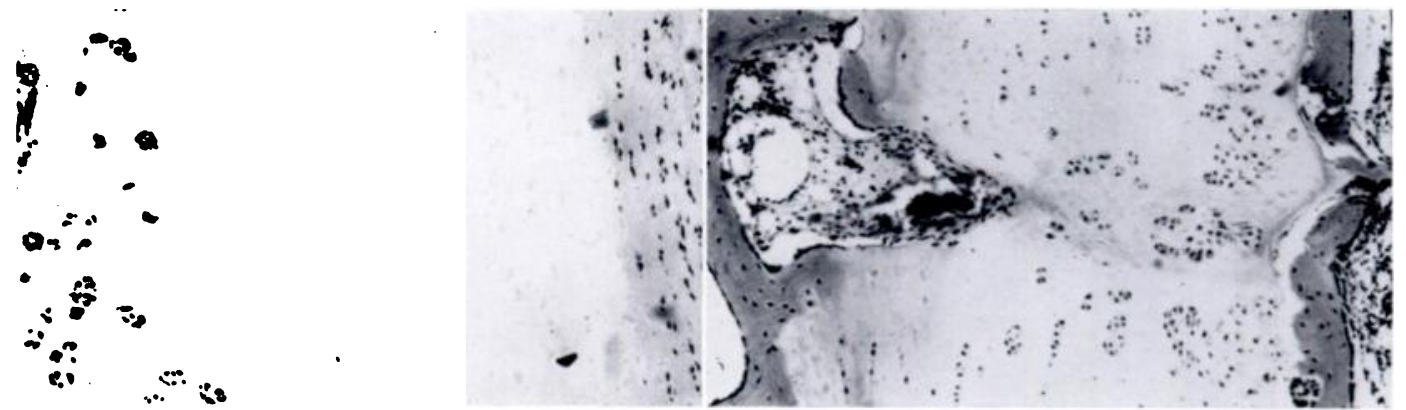

Fici. 5

Fig. 6

Case 1. Figure 1-Initial radiographs. Figure $2-$ Six weeks after operation. Figure 3-One year after operation. Figure 4-Appearance five years after operation on left hip and six years after operation on right hip. There had been no trouble in right hip after operation. Figure 5-Microscopic appearance of articular cartilage taken from femoral head. Figure 6-Microscopic appearance of the epiphysial plate.

was soft, with intense hyperaemia. All tissues were congested. Biopsy specimens of the femoral head and epiphysial plate were taken. The wound healed and the child started to move the hip, but full weight-bearing was avoided for a year. Figure 3 shows the radiographic appearance one year after biopsy of the hip, and Figure 4 shows the appearance five years after operation. The joint space is diminished compared with the opposite hip. Clinically, function was good

VOl. 52 B, NO. 1, FEBRUARY 1970 
at the five-year follow-up, with a flexion range of 0-100 degrees, 30 degrees of abduction and adduction and a normal gait.

Histology-Figure 5 shows the microscopical findings in the articular cartilage of the femoral head and Figure 6 the epiphysial plate. The articular cartilage is thinner than normal. The cartilage at the joint surface contains a layer of flattened eosinophilic fibrous tissue cells not

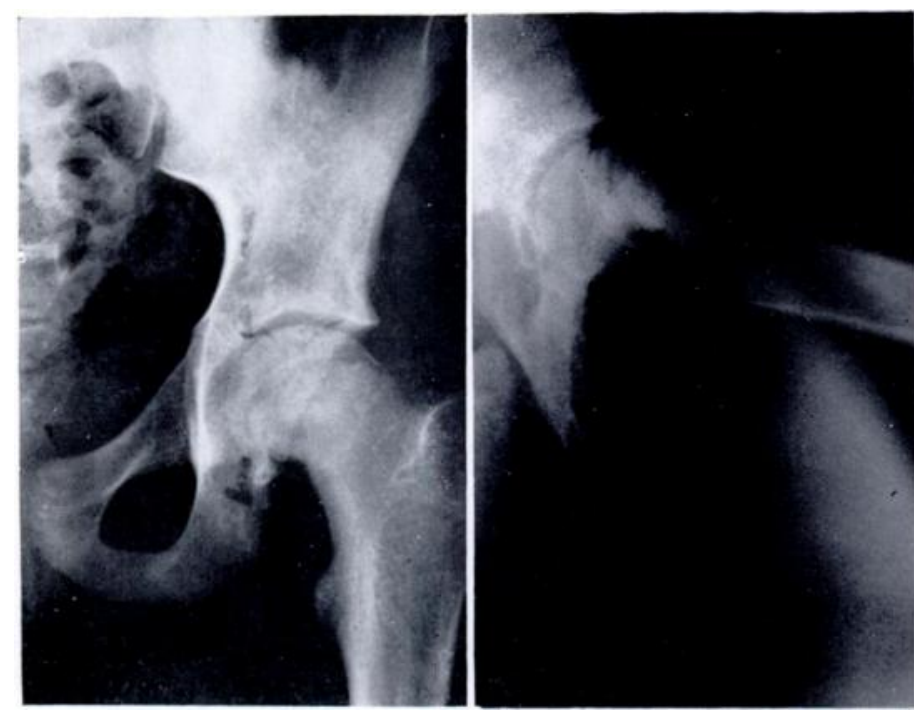

FIG. 7

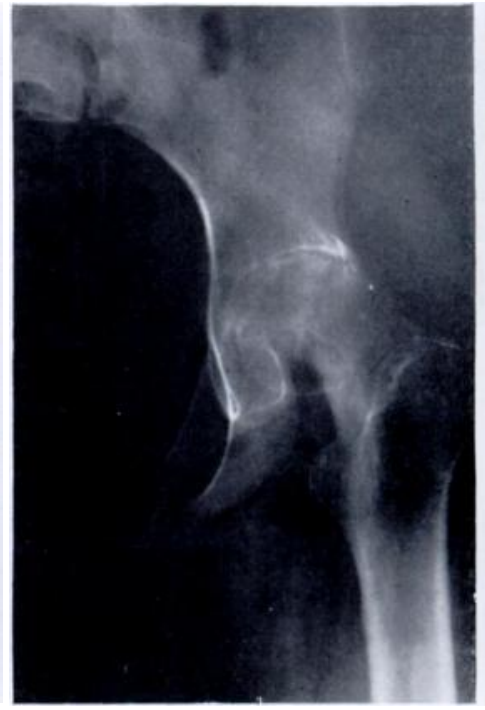

FiG. 8

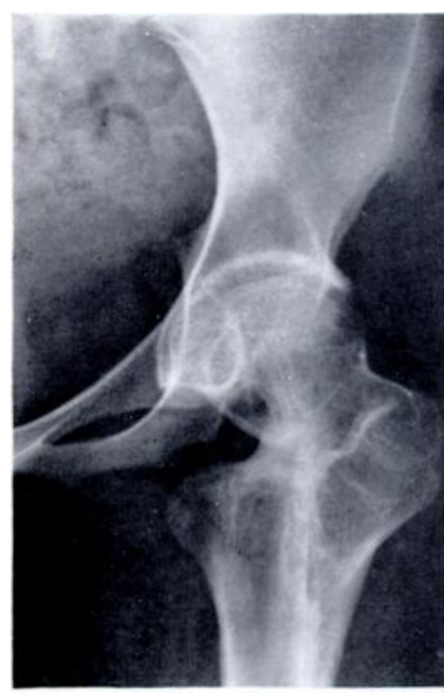

FIci. 9

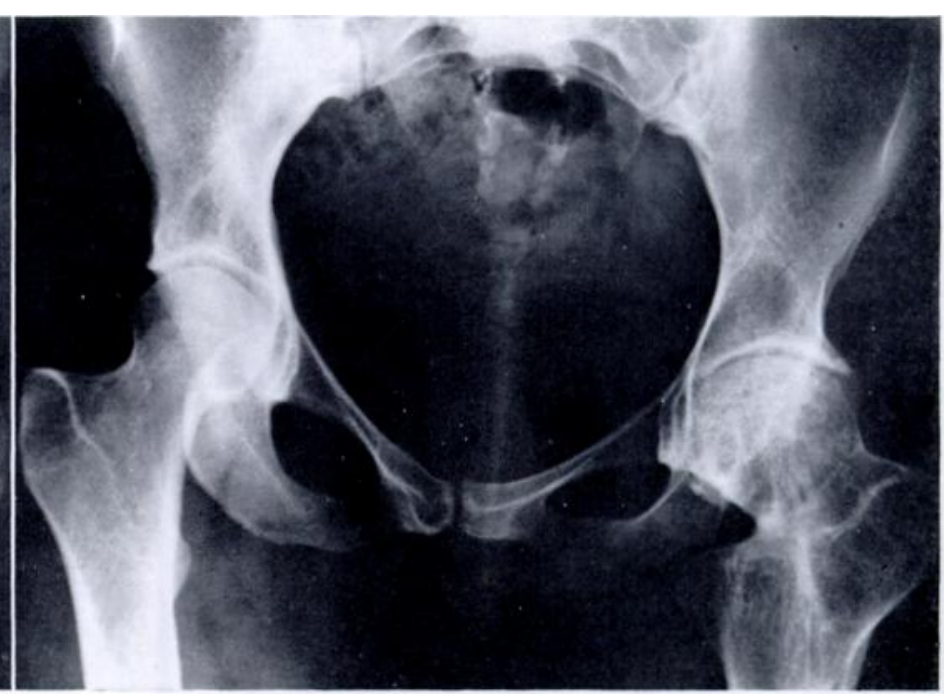

Fig. 10

Case 2. Figure 7-Initial radiographs. Figure 8-One year after first examination. Figure 9-Appearance two years after osteotomy. Figure 10-Sixteen years after osteotomy.

seen in normal articular cartilage. Beneath this is a wide layer without living chondrocytes where the cartilage is necrotic, and a basal layer next to the bony epiphysis where chondrocytes appear viable but show evidence of clumping.

Case 2-A girl aged twelve gave a ten-month history of limp from the left leg. There was loss of medial rotation but other movements were good and there was no spasm. Radiographs at first examination showed considerable slipping of the femoral epiphysis but a good joint space 


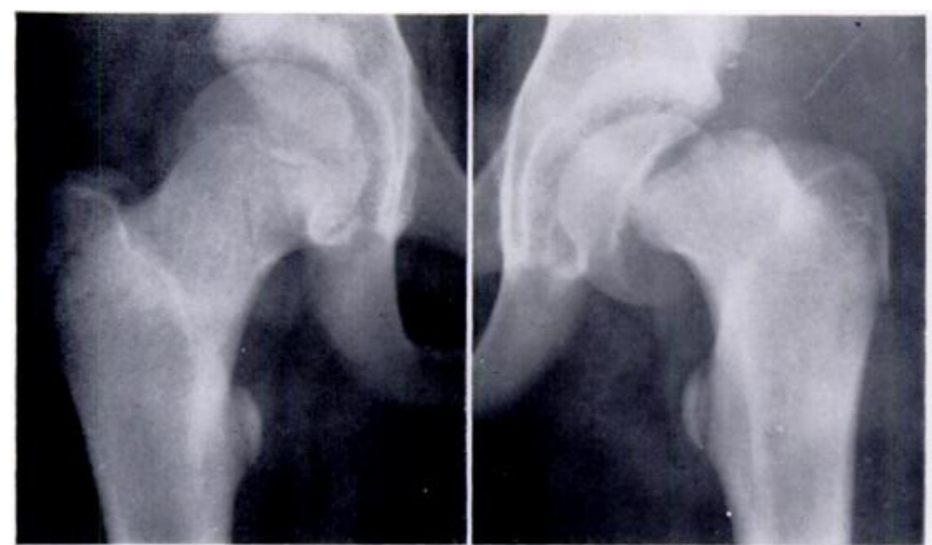

Fig. 11

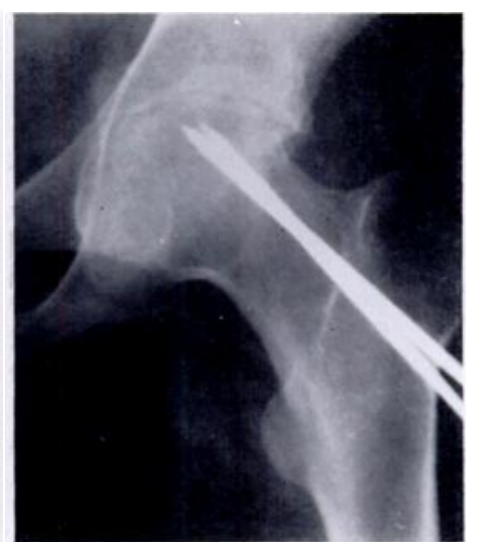

Fig. 12
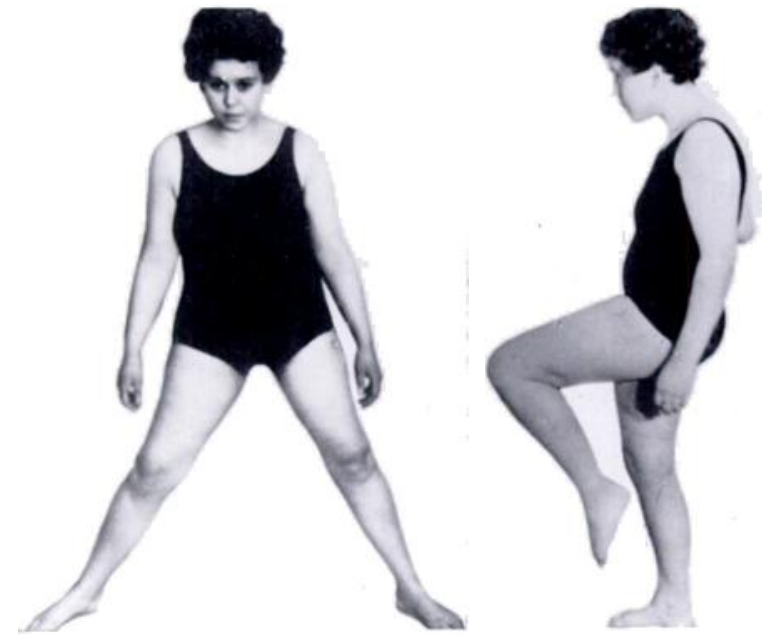

FiG. 13

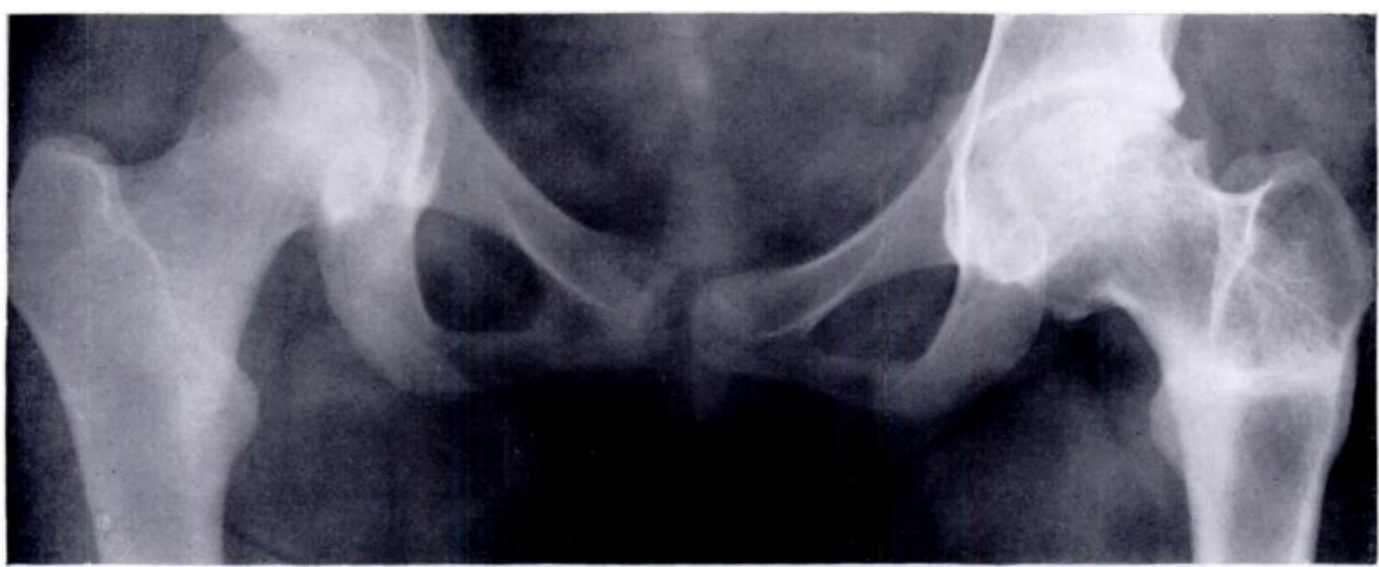

FIG. 14

Case 3. Figure 11--Initial radiograph. Figure 12-Appearance one year after first examination, after epiphysial pinning. Figure 13 - Range of movement at left hip three years after osteotomy. Figure 14 Appearance three years after osteotomy. 
and normal bone texture (Fig. 7). Traction was applied by 9 kilogram weight for two months, and thereafter the hip was protected in a plaster hip spica. She developed a flexion-adduction deformity and movements were much restricted. Radiographs a year after her first attendance showed marked loss of joint space and osteoporosis (Fig. 8).

Operation and progress-Intertrochanteric osteotomy was performed thirteen months from the date of first attendance to correct the contracture and varus deformity. After operation the hip was immobilised on a double abduction frame for three weeks and then by a hip spica for ten weeks. Two years after the osteotomy radiographs showed good recovery of joint space: the femoral head was in good position and the osteotomy was united (Fig. 9). Sixteen years after osteotomy radiographs showed some loss of joint space compared with the radiograph taken fourteen years previously (Fig. 10). Clinical examination at the age of twenty-nine showed a flexion range of 0-60 degrees but no other movement. Function was good; she was able to play badminton, to swim and to dance.

Case 3-A girl aged thirteen presented with a severe slip of the left upper femoral epiphysis but with good joint space and normal bone density (Fig. 11). Manipulation was carried out under general anaesthesia and the epiphysis was secured with two pins. During the six months after operation she developed an abduction contracture and there was progressive loss of movement. Radiographs a year after her first attendance showed marked loss of joint space with osteoporosis (Fig. 12).

Treatment and progress-Traction was applied for two months, after which the pins were removed. The hip was then manipulated under general anaesthesia and a course of exercises was arranged, but without improvement. A second manipulation under general anaesthesia was done but it was not possible to correct the abduction contracture. Intertrochanteric osteotomy was performed to correct the abduction contracture a year and a half after her first attendance. Thereafter the hip was immobilised in a hip spica for six months. After its removal she practised mobilising exercises and gradually recovered movement at the hip (Figs. 13 and 14). Three years after osteotomy she walked well with a slight limp. There was slight discomfort on running but otherwise she has no complaint.

Case 4-A girl aged eleven gave a three months' history of pain in the right hip. Initial radiographs showed considerable posterior displacement of the epiphysis but good joint space and normal bone texture (Fig. 15). Open reduction was done, with internal fixation. Three months later the epiphysis was fusing but there was marked loss of the joint space and porosity of the bone. Figure 16 shows the appearance six months after her first attendance.

Treatment and progress-The adduction contracture was corrected by an intertrochanteric osteotomy about twenty-one months after her first attendance. The joint space gradually returned and she recovered good function (Figs. 17 and 18). She was dancing champion at her school and had walked seventy miles in three days.

Case 5-A boy aged sixteen gave a history of symptoms in the left hip for a year. Radiographs showed considerable posterior slipping of the left femoral epiphysis and slight slipping on the right side (Fig. 19). The joint space on the left was slightly diminished. Open reduction, with a high cervical osteotomy on the epiphysial line laterally but a little distal to the epiphysial line medially, was performed on the left hip, and internal fixation was provided. The right epiphysis was pinned. The osteotomy united within a month of operation (Fig. 20), but there was progressive loss of joint space.

Treatment and progress - The pins were removed because of suspected infection, but they were sterile. Twenty-two months after his first attendance the joint space was shown to be narrow and irregular and the bone porotic. Six years later the joint space had widened again and the surface was smoother (Fig. 21). The range of movement at that time is shown in Figure 22. He complained of slight pain in the hip on first rising and of a feeling of stiffness after activity, but otherwise had good function. 

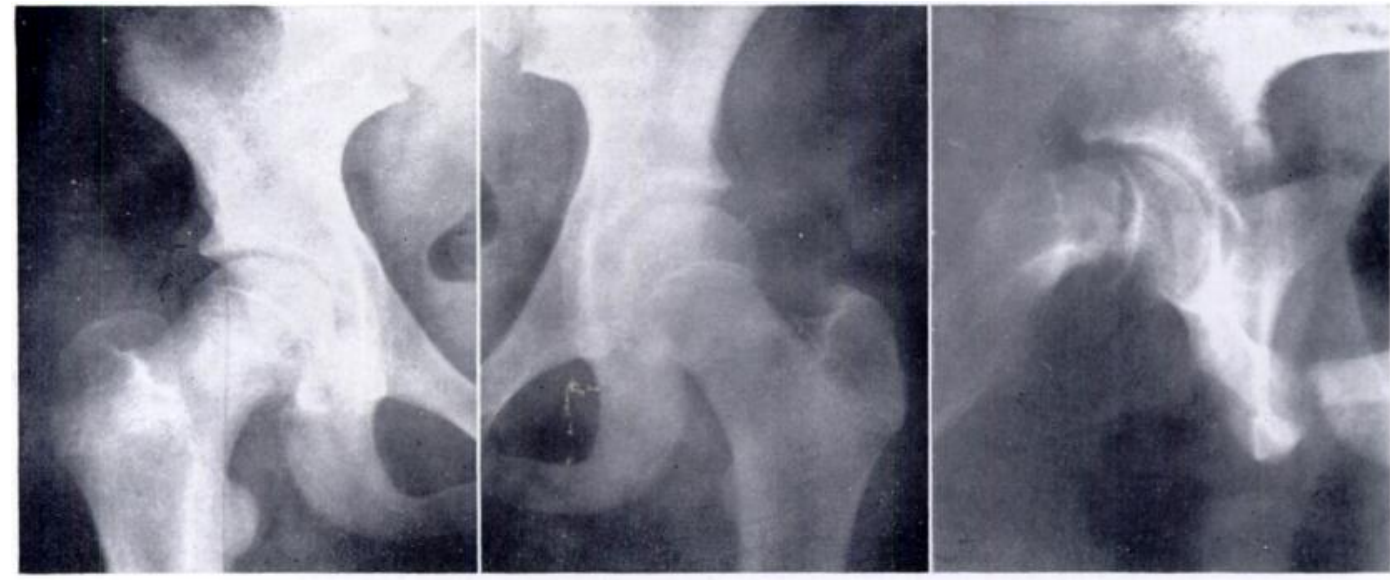

FIG. 15

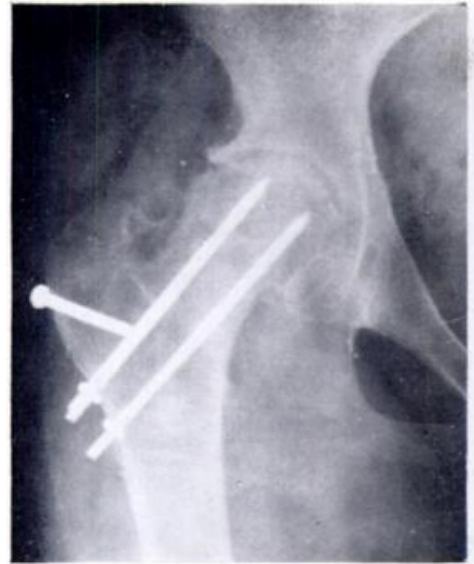

Fici. 16
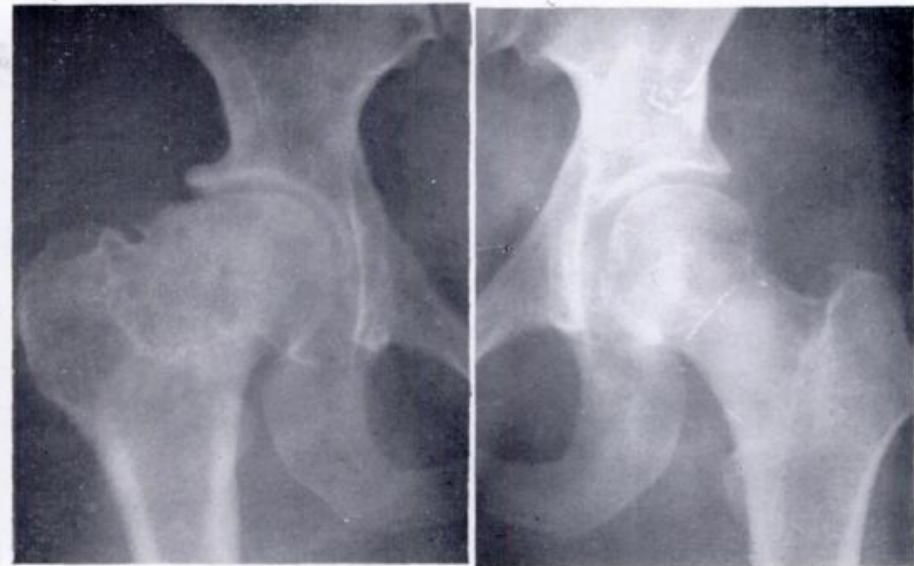

Fig. 17

Case 4. Figure 15-Initial radiographs. Figure 16- Appearance six months after first examination, soon after open reduction. Figure 17-Appearance six years after first examination.

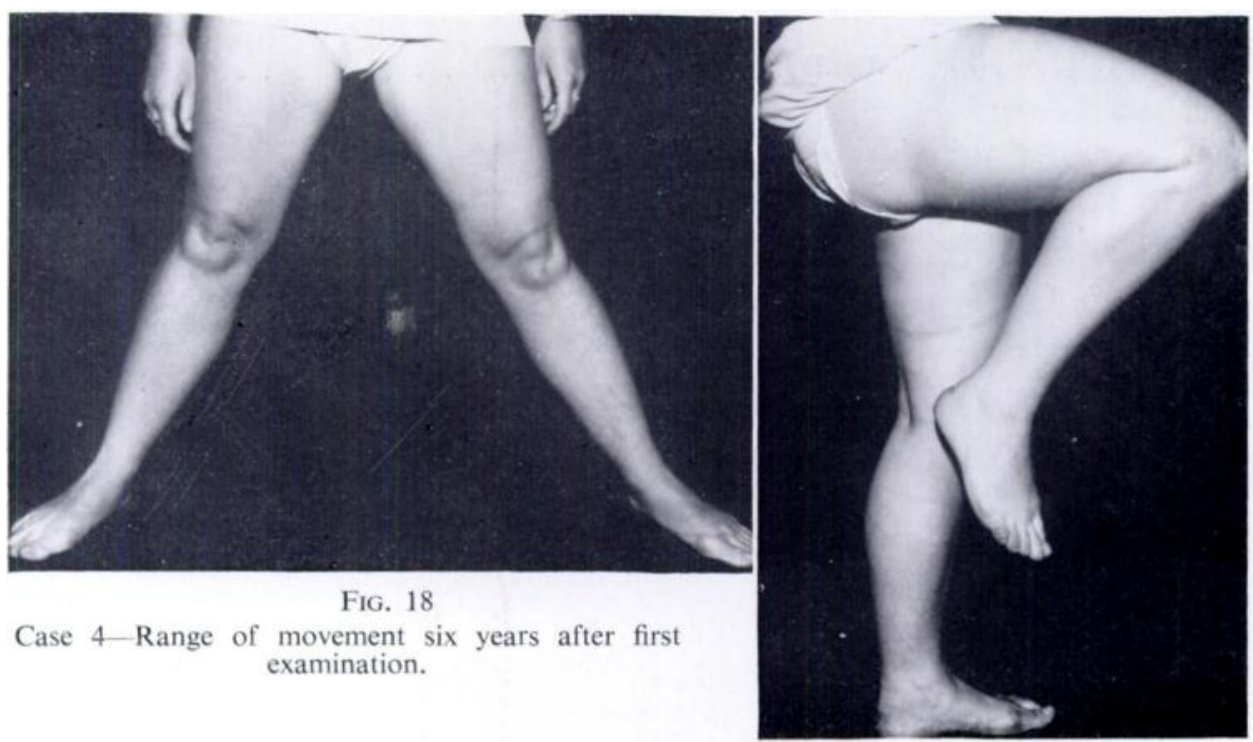

V(I. 52 B, NO. 1, HBRLARY 1970 


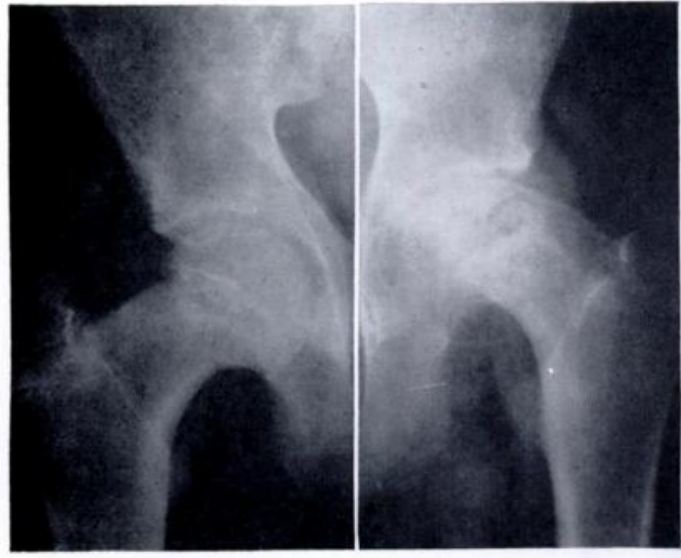

FIG. 19

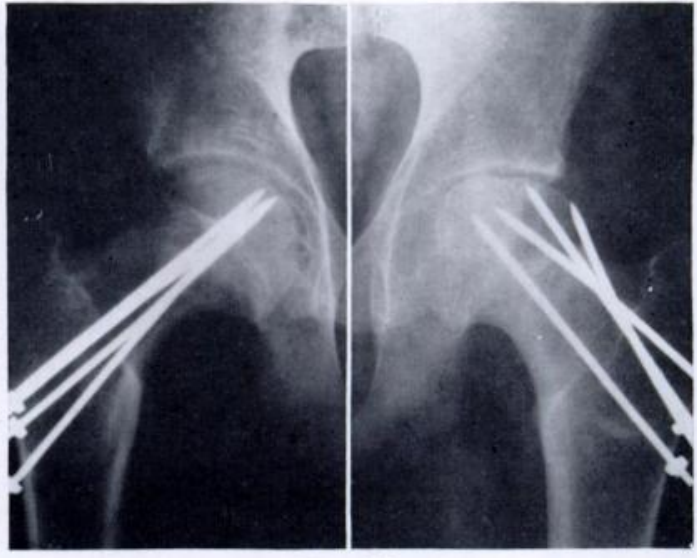

FIG. 20

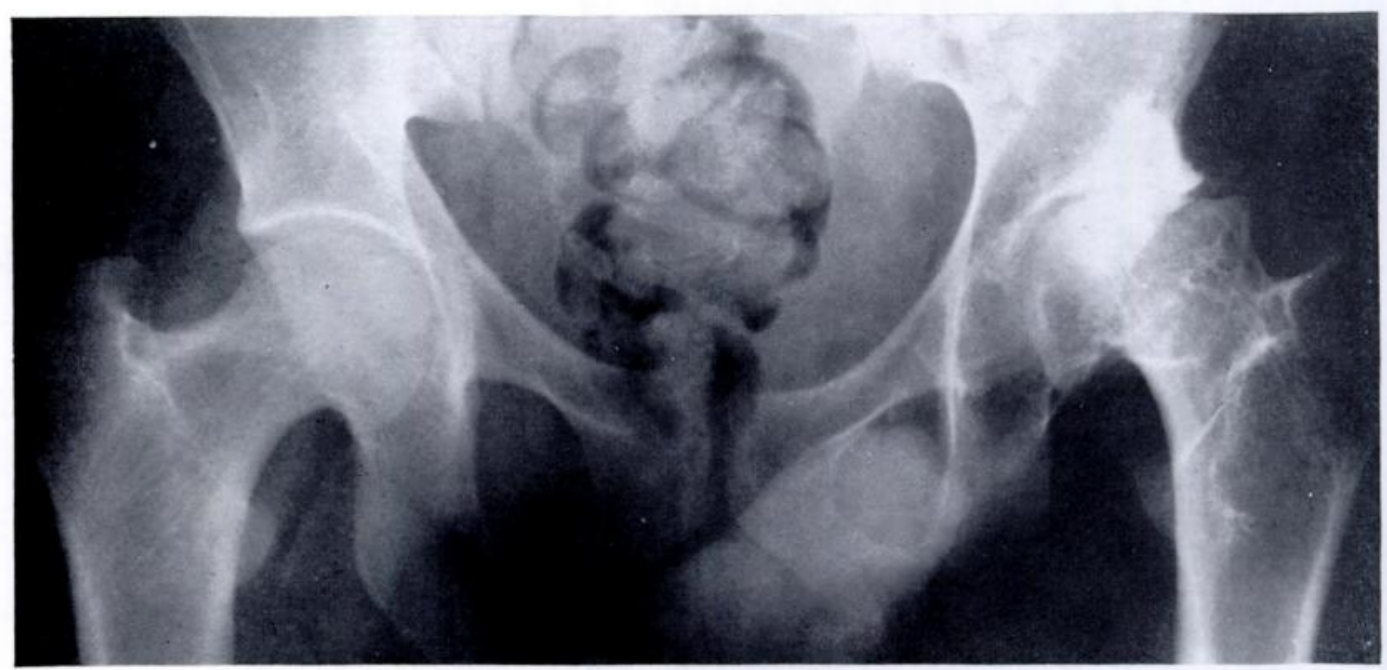

FIG. 21
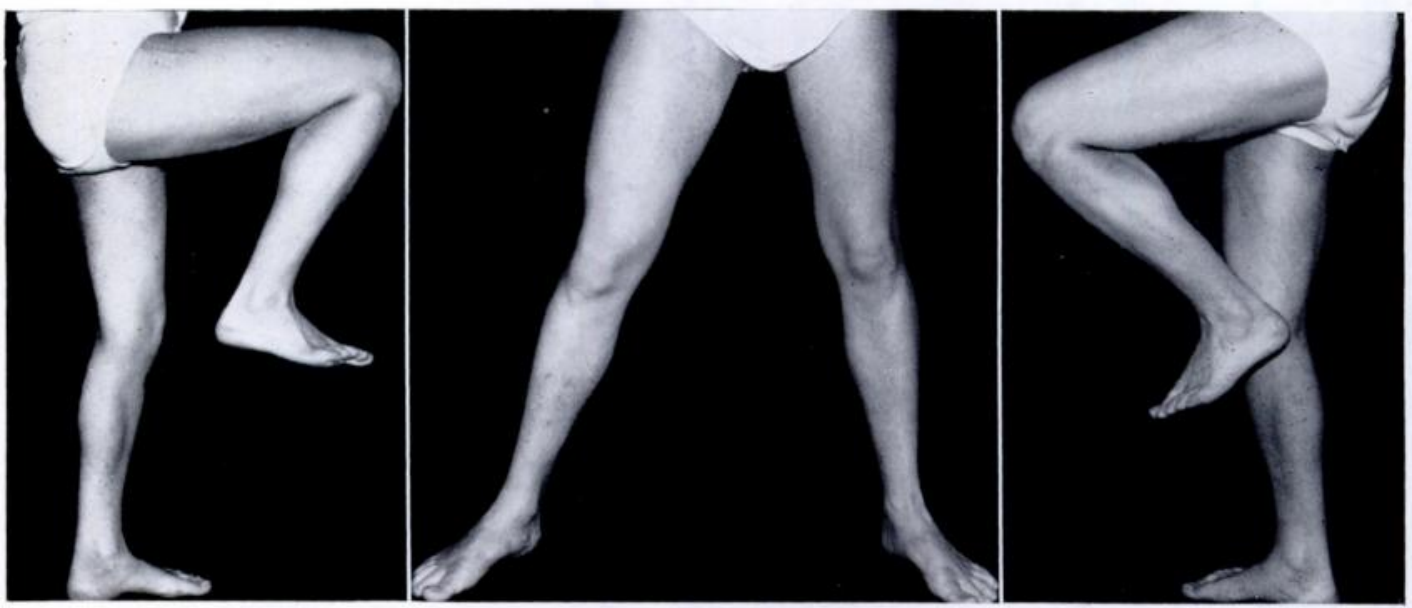

FIG. 22

Case 5. Figure 19-Initial radiograph. Figure 20-Appearance one month after left cervical osteotomy and pinning of right epiphysis. Figure $21-$ Appearance eight years after first presenting. Figure $22-$ Range of movement in hips eight years after first presenting. 


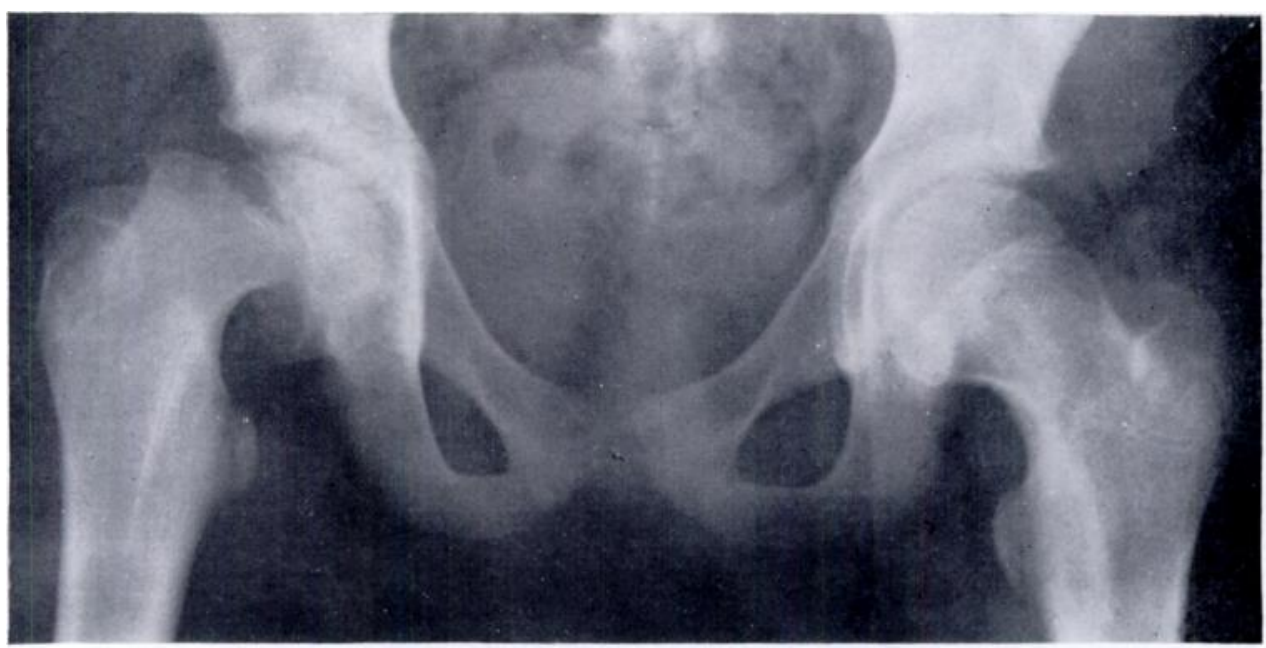

Fici. 23

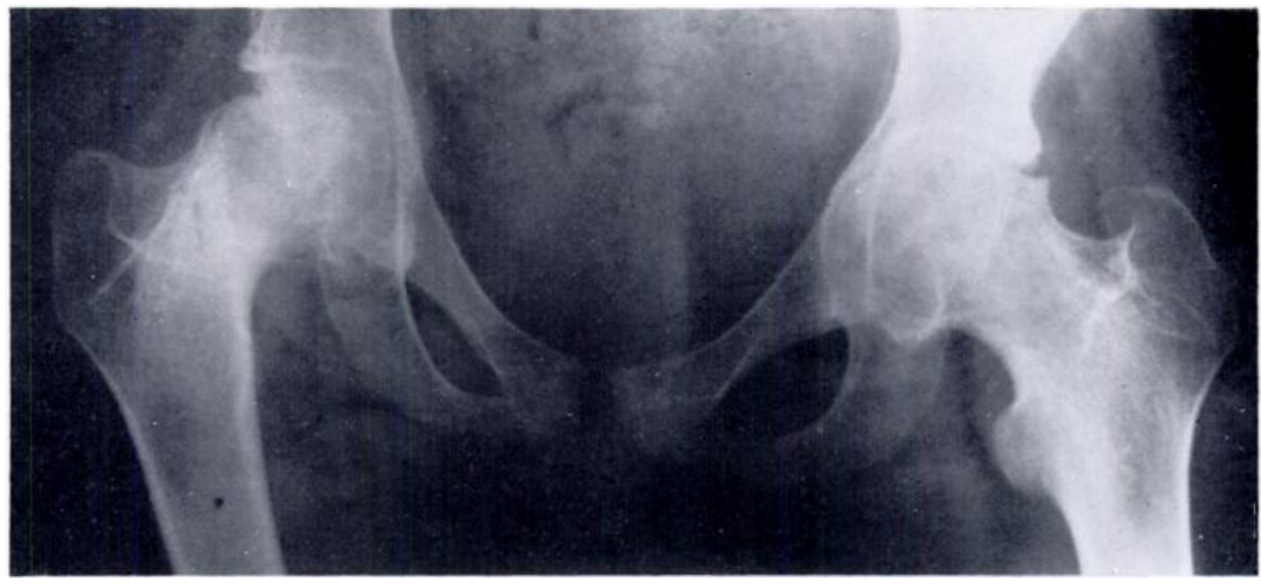

FIG. 24

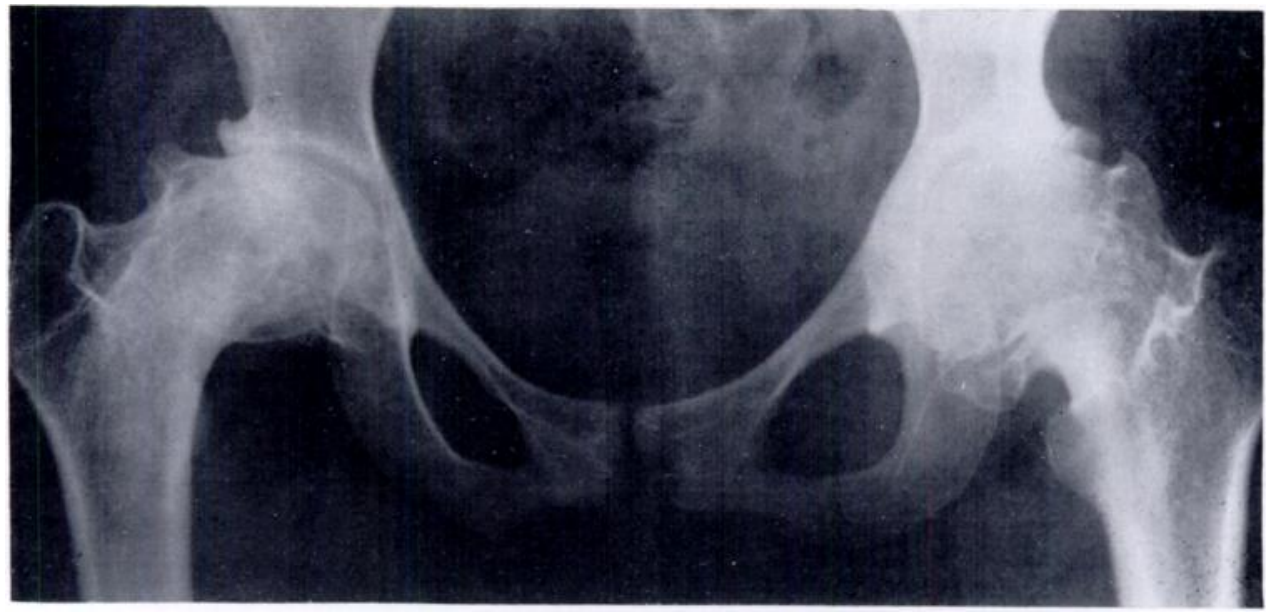

FIG. 25

Case 6. Figure 23-Initial radiograph. Figure 24-Appearance eighteen months after first presenting. Figure 25-Appearance nine years after first presenting.

VOL. 52 B, NO. I, FEBRUARY 1970 
Case 6-A girl aged eleven presented with a four months' history of pain in the right hip and two incidents of falling. The initial radiographs showed severe slipping of the epiphysis on the right side and a slight slip on the left (Fig. 23). The right hip was manipulated under general anaesthesia and a double plaster spica was applied for nine months. After coming out of plaster she had mobilising exercises. Radiographs a year later showed marked loss of joint space in both hips (Fig. 24): there was adduction contracture at the right hip and abduction contracture at the left hip. She walked badly and movements were very restricted. Treatment and progress-She continued exercises for a long time. Figure 25 shows the appearance nine years later and Figure 26 the range of movement then. The contractures have become corrected and there has been some recovery of joint space. Movement is restricted but painless. She is able to lead an active life and does not wish to have further operation.

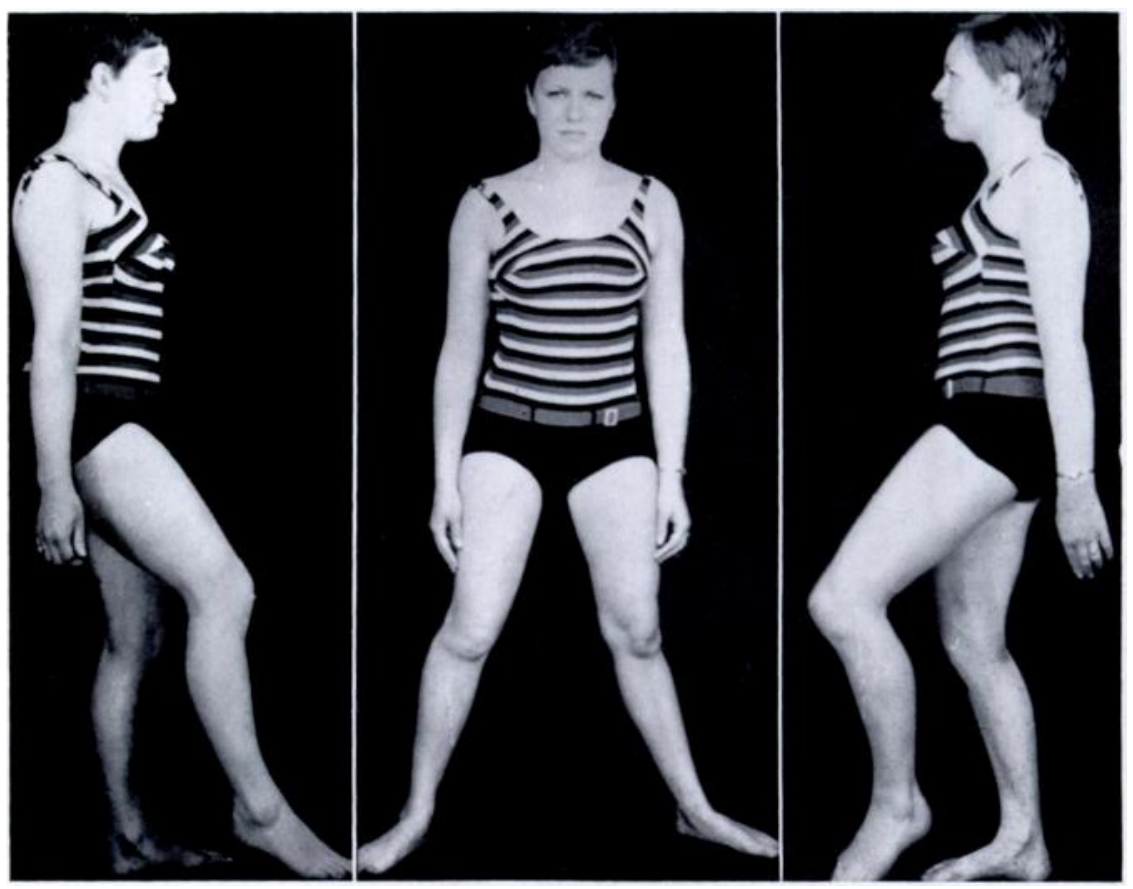

Fig. 26

Case 6-Range of movement of hips nine years after first presenting.

\section{DISCUSSION}

The cause of necrosis of the articular cartilage after slipping of the upper femoral epiphysis remains obscure. Cruess (1963) from a pathological study of two cases in American Negro girls supported the original supposition of Waldenström that necrosis was due to lack of the normal synovial secretion and not to avascular necrosis of the femoral head. The fact that the acetabular changes are indistinguishable from those in the head of the femur make it difficult to ascribe the condition to avascular necrosis of the femoral head alone. Rutishauser and Taillard (1966), however, believed that ischaemia causes a reaction of stasis characterised by capillary retinacular and synovial cell hyperplasia with pannus spreading over the articular cartilage and destruction of the cartilage and consequent fibrous ankylosis. They considered that the mechanism was not specific for necrosis of articular cartilage after slipping of the epiphysis but could be found in other joint conditions in which rheumatoid factors were excluded. 
Pannus formation with destruction of opposing joint surfaces may well be an important factor in some cases of articular cartilage necrosis, but if pannus formation is always a normal response to ischaemia of the femoral head it would be expected to develop in cases of slipped upper femoral epiphysis in which the bone appears avascular as judged from increased radiological density. Several cases of avascular necrosis of the upper femoral epiphysis have been observed but cartilage necrosis has not occurred as would be expected if ischaemia always caused formation of a synovial pannus (Lowe 1961). Similarly, it would be expected to occur in avascular changes of the femoral head after fracture of the neck of the femur in adolescents, but in a large series observed by Ratliff (1967) no case was found. Moreover cartilage necrosis has not been recorded in Perthes' disease, although admittedly this affects younger children.

The source of nutrition of articular cartilage at different ages is uncertain, and our knowledge of the growth and repair of articular cartilage is limited. Experimental work by Ekholm (1955) suggested that there may be some contribution to the nutrition of articular cartilage from the circulation in the subchondral bone, but Ekholm thought that synovial fluid was the main source of nutrition. In Case 1 histological section of the head of the femur (Fig. 5) showed a surviving layer of basal chondrocytes and death of the superficial two-thirds of the articular cartilage. It seems that necrosis of the superficial two-thirds of the articular cartilage may have resulted from loss of synovial secretion while the surviving basal layer received its nutrition from the adjacent bony epiphysis. Survival of the basal layer suggests that the blood supply to the bony epiphysis was intact and that cartilage necrosis is not due to avascular necrosis of the bony epiphysis.

These cases demonstrate that a degree of recovery from articular cartilage necrosis can occur, probably from survival of the basal layer of the articular cartilage nourished from the bony epiphysis, and with a slow return of synovial secretion the cells are able to survive as they proliferate further from the basal layer of chondrocytes. Whether chondrocytes in the articular cartilage can divide and grow new cells is not known. It may happen in children but not in adults. The improved joint space in the cases showing recovery may be due to a formation of a crude fibrocartilage rather than new hyaline cartilage.

If there is a combination of avascular necrosis of the bony epiphysis and loss of the synovial secretion then recovery of articular cartilage would be unlikely, and this may account for the poor prognosis in some cases of articular cartilage necrosis. From these cases it is evident that the prognosis after articular cartilage necrosis is not inevitably poor; so arthrodesis or arthroplasty should not be precipitate. With patience and prolonged non-weight-bearing exercises, and with correction of established contracture, a number of patients may gain reasonable recovery.

\section{SUMMARY}

1. Six cases of necrosis of articular cartilage complicating slipping of the upper femoral epiphysis are reviewed: histological examination in one case showed death of the superficial two-thirds of the articular cartilage, with survival of a layer of basal chondrocytes. In all six cases, after severe initial reduction of joint space as seen radiographically, there was gradual return of joint space, suggesting some regeneration of articular cartilage. The prognosis after cartilage necrosis is therefore not always so bad as has been supposed.

2. Various hypotheses concerning the cause of cartilage necrosis complicating slipped epiphysis are reviewed. The precise cause remains unknown, but there is substantial evidence against its being a consequence of ischaemia of the femoral head.

I am most grateful to Mr N. J. Blockey, Mr D. M. Dunn and Mr D. Wainwright for allowing me to study Cases 1, 2 and 5, and 4 respectively. The illustrations have been prepared by $\mathrm{Dr} \mathrm{L}$. Bowcock of the Department of Medical Illustration, North Staffordshire Royal Infirmary.

VOL. 52 B, NO. 1, FFBRUARY 1970 


\section{REFERENCES}

Cruess, R. L. (1963): The Pathology of Acute Necrosis of Cartilage in Slipping of the Capital Femoral Epiphysis. Journal of Bone and Joint Surgery, 45-A, 1013.

Eкноцм, R. (1955): Nutrition of Articular Cartilage. Acta Anatomica, 24, 329.

Elmslif, R. C. (1913): Coxa Vara, its Pathology and Treatment. London: Henry Frowde, and Hodder \& Stoughton, and in Transactions of the International Congress of Medicine, London 1913 (1914, Sub-section VII (a), Orthopaedics, pt. 2. 41).

Lowe, H. G. (1961): Avascular Necrosis after Slipping of the Upper Femoral Epiphysis. Journal of Bone and Joint Surgery, 43-B, 688.

Ratliff, A. H. C. (1967): Personal communication.

Rutishauser, E., and TAillard, W. (1966): L'ischémie articulaire en pathologie humaine et expérimentale. La notion de pannus vasculaire. Revue de chirurgie orthopédique et réparatrice de l'appareil moteur, 52, 197.

Waldenström, H. (1930): On Necrosis of the Joint Cartilage by Epiphyseolysis Capitis Femoris. Acta chirurgica Scandinavica, 67, 936. 